\title{
Smarandache Curves According to Sabban Frame of the anti-Salkowski Indicatrix Curve
}

\author{
Süleyman Şenyurt ${ }^{1 *}$ and Burak Öztürk ${ }^{1}$ \\ ${ }^{1}$ Department of Mathematics, Faculty of Science and Arts, Ordu University, Ordu, Turkey \\ *Corresponding author E-mail: senyurtsuleyman@hotmail.com
}

\section{Article Info}

Keywords: Anti-Salkowski curve, Sabban frame, Smarandache curves

2010 AMS: $53 A 04$

Received: 20 July 2019

Accepted: 05 November 2019

Available online: 20 December 2019

\begin{abstract}
The aim of this paper is to define Smarandache curves according to the Sabban frame belonging to the spherical indicatrix curve of the anti-Salkowski curve. We also illustrate these curves with the Maple program and calculate the geodesic curvatures of these curves.
\end{abstract}

\section{Introduction}

Erich Salkowski (1881-1943), a German mathematician. In 1909, he defined curve families with non-constant $\tau$ and constant curvature $\kappa$ [1]. Later J. Monterde constructed a method for closed curves and the properties of anti-Salkowski curve used in [2]. For authors worked on the anti-Salkowski curve also can be seen in [3]-[7]. When the Frenet vectors of any curve are taken as the position vector, then the regular curves generated by these vectors are called Smarandache curves [8]. Smarandache curves in Euclidean 3-space are defined and some features of these curves are given in [9]. For some authors worked on the Smarandache curve also may be seen in [10, 11]. In 1990, the geodesic curve of a spherical curve is calculated by J. Koenderink with the Sabban frame of the spherical indicatrix curves in [12]. Then the Smarandache curves obtained from Sabban frame are defined and geodesic curvatures of these curves are given in [13].

In this study, Smarandache curves are defined according to the Sabban frames belonging to the spherical indicatrix curves of each of the $T, N, B$ Frenet vectors of the anti-Salkowski curve. The geodesic curvatures of these curves are then calculated.

\section{Preliminaries}

In the Euclidean 3-space $E^{3}$, the Frenet frame of any curve $\alpha$ is given by $\{T, N, B\}$. For an arbitrary curve $\alpha \in E^{3}$, with the first and second curvatures, $\kappa$ and $\tau$ respectively, the Frenet apparatus are given by [14]

$$
T^{\prime}=\kappa N, \quad N^{\prime}=-\kappa T+\tau B, \quad B^{\prime}=-\tau N .
$$

Accordingly, the spherical indicatrix curves of Frenet vectors are $(T),(N)$ and $(B)$ respectively.These equations of curves are given by [14]

$$
\alpha_{T}(s)=T(s), \quad \alpha_{N}(s)=N(s), \quad \alpha_{B}(s)=B(s) .
$$

Let $\gamma: I \rightarrow S^{2}$ be a unit speed spherical curve. We denote $s$ as the arc-length parameter of $\gamma$. Let us denote by [14]

$$
\gamma(s)=\gamma(s), \quad t(s)=\gamma^{\prime}(s), \quad d(s)=\gamma(s) \wedge t(s) .
$$

We call $t(s)$ a unit tangent vector of $\gamma .\{\gamma, t, d\}$ frame is called the Sabban frame of $\gamma$ on $S^{2}$. Then we have the following spherical Frenet formulae of $\gamma$ :

$$
\gamma^{\prime}=t, \quad t^{\prime}=-\gamma+\kappa_{g} d, \quad d^{\prime}=-\kappa_{g} t
$$

Email addresses and ORCID numbers: senyurtsuleyman@hotmail.com, https://orcid.org/0000-0003-1097-5541 (S. Şenyurt), brkztrk4152@gmail.com, https://orcid.org/0000-0001-9998-4924 (B. Öztürk) 
where is called the geodesic curvature of $\kappa_{g}$ on $S^{2}$ and

$$
\kappa_{g}=\left\langle t^{\prime}, d\right\rangle
$$

$[12,13]$.

Definition 2.1. (anti-Salkowski curve) [2]. For any $m \in \mathbb{R}$ with $m \neq \mp \frac{1}{\sqrt{3}}, 0$, let us define the space curve

$$
\begin{aligned}
\beta_{m}(s)= & \left(\frac{n}{2\left(4 n^{2}-1\right) m}\left(n\left(1-4 n^{2}+3 \cos (2 n s)\right) \cos (s)+\left(2 n^{2}+1\right) \sin (s) \sin (2 n s)\right),\right. \\
& \left.\frac{n}{2\left(4 n^{2}-1\right) m}\left(n\left(1-4 n^{2}+3 \cos (2 n s)\right) \sin (s)-\left(2 n^{2}+1\right) \cos (s) \sin (2 n s), \frac{n^{2}-1}{4 n}(2 n s+\sin (2 n s))\right)\right)
\end{aligned}
$$

where $n=\frac{m}{\sqrt{1+m^{2}}}$. The Frenet apparatus are

$$
\left\{\begin{array}{l}
\kappa \\
T(s)=-\left(\cos (s) \sin (n s)-n \sin (s) \cos (n s), \sin (s) \sin (n s)+n \cos (s) \cos (n s), \frac{n}{m} \cos (n s)\right), \\
N(s)=n\left(\frac{\sin (s)}{m},-\frac{\cos (s)}{m}, 1\right), \\
B(s)=\left(-\cos (s) \cos (n s)-n \sin (s) \sin (n s),-\sin (s) \cos (n s)+n \cos (s) \sin (n s), \frac{n}{m} \sin (n s)\right) .
\end{array}\right.
$$

The shape of this curve is given in Figure (2.1)

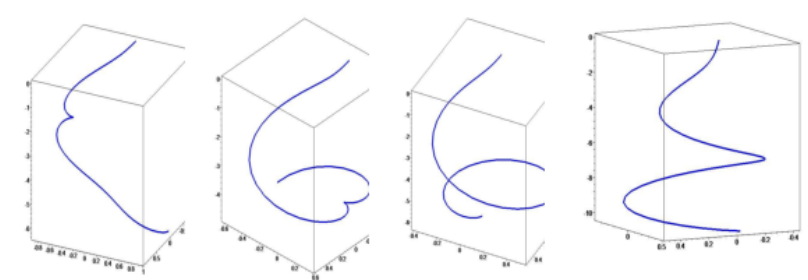

Figure 2.1: anti-Salkowski Curve, $m=\frac{1}{3}, \frac{1}{5}, \frac{1}{8}, \frac{1}{16}$ and $s=[-5,5]$

Let $(\alpha),(\delta)$ and $(\zeta)$ be spherical indicatrix curves of tangent, principal normal and binormal vectors belonging to anti-Salkowski curve, respectively. Using the equations (2.1) and (2.2), Sabban apparatus belonging to these curves is given by

$$
\begin{gathered}
T=T, \quad T_{T}=N, \quad T \wedge T_{T}=B, \\
T^{\prime}=T_{T}, \quad T_{T}^{\prime}=-T+\frac{1}{\tan (n s)}\left(T \wedge T_{T}\right), \quad\left(T \wedge T_{T}\right)^{\prime}=-\frac{1}{\tan (n s)} T_{T}, \\
K_{g}^{T}=\frac{1}{\kappa}=\frac{1}{\tan (n s)} . \\
T(s)=\left(\cos (s) \sin (n s)-n \sin (s) \cos (n s), \sin (s) \sin (n s)+n \cos (s) \cos (n s), \frac{n}{m} \cos (n s)\right), \\
T_{T}(s)=n\left(\frac{\sin (s)}{m},-\frac{\cos (s)}{m}, 1\right),
\end{gathered}
$$$$
\left(T \wedge T_{T}\right)(s)=-\left(\cos (s) \cos (n s)+n \sin (s) \sin (n s), \sin (s) \cos (n s)-n \cos (s) \sin (n s), \frac{n}{m} \sin (n s)\right) .
$$$$
N=N, \quad T_{N}=\frac{-\tan (n s) T+B}{\sqrt{\tan ^{2}(n s)+1}}, \quad N \wedge T_{N}=\frac{T+\tan (n s) B}{\sqrt{\tan ^{2}(n s)+1}},
$$$$
N^{\prime}=T_{N}, \quad T_{N}^{\prime}=\frac{\tan (n s)^{\prime}}{\sqrt{\tan ^{2}(n s)+1}} N+N \wedge T_{N}, \quad\left(T \wedge T_{T}\right)^{\prime}=\frac{-\tan (n s)^{\prime}}{\sqrt{\tan ^{2}(n s)+1}} T_{N},
$$$$
K_{g}^{N}=\frac{-\kappa^{\prime}}{\sqrt{\kappa^{2}+1}}=\frac{-\tan (n s)^{\prime}}{\sqrt{\tan ^{2}(n s)+1}} .
$$ 


$$
\begin{aligned}
& N(s)=\left(\frac{n \sin (s)}{m},-\frac{n \cos (s)}{m}, n\right) \\
& T_{N}(s)=\frac{1}{\sqrt{\tan ^{2}(n s)+1}}(-\cos (s) \cos (n s)-n \sin (s) \sin (n s)-\tan (n s)(-\cos (s) \sin (n s)+n \sin (s) \cos (n s)), \\
& \left.-\tan (n s)(-\sin (s) \sin (n s)-n \cos (s) \cos (n s))-\sin (s) \cos (n s)+n \cos (s) \sin (n s), \frac{2 n}{m} \sin (n s)\right), \\
& \left(N \wedge T_{N}\right)(s)=\frac{1}{\sqrt{\tan ^{2}(n s)+1}}(\tan (n s)(-\cos (s) \cos (n s)-n \sin (s) \sin (n s))-\cos (s) \sin (n s)+n \sin (s) \cos (n s),-\sin (s) \sin (n s) \\
& \left.+\tan (n s)(-\sin (s) \cos (n s)+n \cos (s) \sin (n s))-n \cos (s) \cos (n s), \frac{n}{m} \tan (n s) \sin (n s)-\frac{n}{m} \cos (n s)\right) . \\
& B=B, \quad T_{B}=-N, \quad B \wedge T_{B}=T, \\
& B^{\prime}=T_{B}, \quad B_{T}^{\prime}=-B+\tan (n s)\left(B \wedge T_{B}\right) \\
& \left(B \wedge T_{B}\right)^{\prime}=\tan (n s) T_{B}, \quad K_{g}^{B}=\kappa=\tan (n s) . \\
& B(s)=-\left(\cos (s) \cos (n s)+n \sin (s) \sin (n s), \sin (s) \cos (n s)-n \cos (s) \sin (n s), \frac{n}{m} \sin (n s)\right), \\
& T_{B}(s)=-\left(\frac{n \sin (s)}{m},-\frac{n \cos (s)}{m}, n\right) \\
& \left(B \wedge T_{B}\right)(s)=-\left(\cos (s) \sin (n s)-n \sin (s) \cos (n s), \sin (s) \sin (n s)+n \cos (s) \cos (n s),-\frac{n}{m} \cos (n s)\right) .
\end{aligned}
$$

\section{Smarandache curves according to the Sabban frame belonging to spherical indicatrix curve of the anti-Salkowski curve}

Definition 3.1. Let $\alpha=\alpha(s)$ be a curve and $\left\{T, T_{T}, T \wedge T_{T}\right\}$ be Sabban frame of this curve. Then $T T_{T}$-Smarandache curve is given by

$$
\alpha_{1}(s)=\frac{1}{\sqrt{2}}\left(T+T_{T}\right)
$$

According to equation (2.4) we can parameterize the $\alpha_{1}(s)$-Smarandache curve as in the following form

$$
\alpha_{1}(s)=\frac{1}{\sqrt{2}}\left(-\cos (s) \sin (n s)+n \sin (s) \cos (n s)+\frac{n}{m} \sin (s),-\sin (s) \sin (n s)-n \cos (s) \cos (n s)-\frac{n}{m} \cos (s),-\frac{n}{m} \cos (n s)+n\right) .
$$

The shape of this curve is given in Figure (3.1)
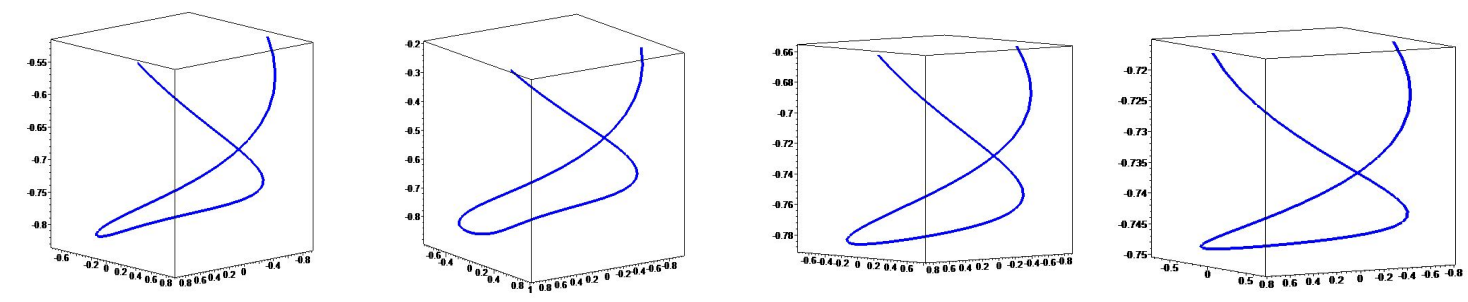

Figure 3.1: $T T_{T}$-Smarandache Curve, $m=\frac{1}{3}, \frac{1}{5}, \frac{1}{8}, \frac{1}{16}$ and $s=[-5,5]$

Theorem 3.2. The geodesic curvature $K_{g}^{\alpha_{1}}$ according to $\alpha_{1}(s)$-Smarandache curve is

where the coefficients $\chi_{1}, \chi_{2}$ and $\chi_{3}$ are

$$
K_{g}^{\alpha_{1}}=\frac{\tan ^{4}(n s)}{(2 \tan (n s)+1)^{\frac{5}{2}}}\left(\chi_{1}-\chi_{2}+2 \tan (n s) \chi_{3}\right),
$$

$$
\begin{aligned}
& \chi_{1}=-2-\frac{1}{\tan ^{2}(n s)}+\frac{1}{\tan (n s)}\left(\frac{1}{\tan (n s)}\right)^{\prime}, \\
& \chi_{2}=-2-\frac{1}{\tan (n s)}\left(\frac{1}{\tan (n s)}\right)^{\prime}-\frac{3}{\tan ^{2}(n s)}-\frac{1}{\tan ^{4}(n s)}, \\
& \chi_{3}=\frac{2}{\tan (n s)}+\left(\frac{2}{\tan (n s)}\right)^{\prime}+\frac{1}{\tan ^{3}(n s)} .
\end{aligned}
$$


Proof. If we take the derivative of (3.1) and from the equation (2.3) we get

$$
\left(T_{T}\right)_{\alpha_{1}} \cdot \frac{d s^{*}}{d s}=\frac{1}{\sqrt{2}}\left(-T+T_{T}+\frac{1}{\tan (n s)}\left(T \wedge T_{T}\right)\right),
$$

if we take the norm of (3.2) we have

$$
\frac{d s^{*}}{d s}=\frac{\sqrt{2 \tan ^{2}(n s)+1}}{\tan (n s) \sqrt{2}} .
$$

We obtain the tangent of $\alpha_{1}(s)$-Smarandahce curve as in

$$
\left(T_{T}\right)_{\alpha_{1}}=\frac{1}{\sqrt{2 \tan ^{2}(n s)+1}}\left(-\tan (n s) T+\tan (n s) T_{T}+\left(T \wedge T_{T}\right)\right) .
$$

The derivative of (3.2) is

$$
\left(T_{T}\right)_{\alpha_{1}}^{\prime}=\frac{1}{\sqrt{2 \tan ^{2}(n s)+1}}\left(\chi_{1} T+\chi_{2} T_{T}+\chi_{3}\left(T \wedge T_{T}\right)\right) .
$$

From equations (3.1) and (3.3) we have

$$
\left(T \wedge T_{T}\right)_{\alpha_{1}}=\frac{1}{\sqrt{2 \tan ^{2}(n s)+1}}\left(T-T_{T}+2 \tan (n s)\left(T \wedge T_{T}\right)\right) .
$$

So the geodesic curvature from the equation (2.3) is

$$
K_{g}^{\alpha_{1}}=\frac{\tan ^{4}(n s)}{(2 \tan (n s)+1)^{\frac{5}{2}}}\left(\chi_{1}-\chi_{2}+2 \tan (n s) \chi_{3}\right) .
$$

Definition 3.3. Let $\alpha=\alpha(s)$ be a curve and $\left\{T, T_{T}, T \wedge T_{T}\right\}$ be Sabban frame of this curve. Then $T\left(T \wedge T_{T}\right)$-Smarandache curve is given by

$$
\alpha_{2}(s)=\frac{1}{\sqrt{2}}\left(T+\left(T \wedge T_{T}\right)\right) .
$$

According to equation (2.4) we can parameterize the $\alpha_{2}(s)$-Smarandache curve as in the following form

$$
\begin{aligned}
\alpha_{2}(s)= & \frac{1}{\sqrt{2}}(-\cos (s)(\cos (n s)-\sin (n s))+n \sin (s)(\cos (n s)+\sin (n s)), \\
& \left.\sin (s)(\cos (n s)-\sin (n s))-n \cos (s)(\cos (n s)+\sin (n s)),-\frac{n}{m}(\cos (n s)+\sin (n s))\right) .
\end{aligned}
$$

The shape of this curve is given in Figure (3.2)
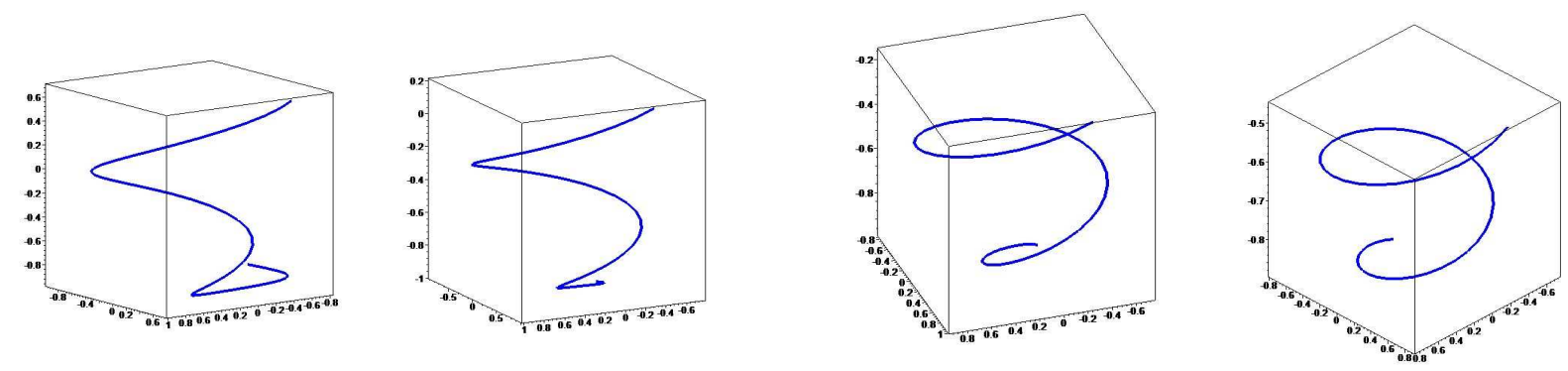

Figure 3.2: $T\left(T \wedge T_{T}\right)$-Smarandache Curve, $m=\frac{1}{3}, \frac{1}{5}, \frac{1}{8}, \frac{1}{16}$ and $s=[-5,5]$

Theorem 3.4. The geodesic curvature $K_{g}^{\alpha_{2}}$ according to $\alpha_{2}(s)$-Smarandache curve is given by

$$
K_{g}^{\alpha_{2}}=\frac{\tan (n s)+1}{\tan (n s)} .
$$

Proof. If we take the derivative of (3.4) and from the equation (2.3) we get,

$$
\left(T_{T}\right) \alpha_{2} \cdot \frac{d s^{*}}{d s}=\frac{1}{\sqrt{2}}\left(T_{T}-\frac{1}{\tan (n s)} T_{T}\right)
$$

if we take the norm of (3.5), $\frac{d s^{*}}{d s}=\frac{\tan (n s)-1}{\tan (n s) \sqrt{2}}$ we have, We obtain the tangent of $\alpha_{2}(s)$-Smarandahce curve as in

$$
\left(T_{T}\right)_{\alpha_{2}}=T_{T}
$$


The derivative in the (3.6) is

$$
\left(T_{T}\right)_{\alpha_{2}}^{\prime} \cdot \frac{d s^{*}}{d s}=\frac{\sqrt{2}}{\tan (n s)-1}\left(-\tan (n s) T+\left(T \wedge T_{T}\right)\right) .
$$

From equations (3.4) and (3.6) we have

$$
\left(T \wedge T_{T}\right)_{\alpha_{2}}=\frac{1}{\sqrt{2}}\left(-T+\left(T \wedge T_{T}\right)\right) .
$$

So the geodesic curvature from the equation (2.3) is

$$
K_{g}^{\alpha_{2}}=\frac{\tan (n s)+1}{\tan (n s)} .
$$

Definition 3.5. Let $\alpha=\alpha(s)$ be a curve and $\left\{T, T_{T}, T \wedge T_{T}\right\}$ be Sabban frame of this curve. Then $T_{T}\left(T \wedge T_{T}\right)$-Smarandache curve is given by

$$
\alpha_{3}(s)=\frac{1}{\sqrt{2}}\left(T_{T}+\left(T \wedge T_{T}\right)\right)
$$

According to equation (2.4) we can parameterize the $\alpha_{4}(s)$-Smarandache curve as in the following form

$$
\alpha_{3}(s)=\frac{1}{\sqrt{2}}\left(\cos (s) \cos (n s)+n \sin (s) \sin (n s)+\frac{n}{m} \sin (s), \sin (s) \cos (n s)-n \cos (s) \sin (n s)-\frac{n}{m} \cos (s),-\frac{n}{m} \sin (n s)+n\right) .
$$

The shape of this curve is given in Figure (3.3)
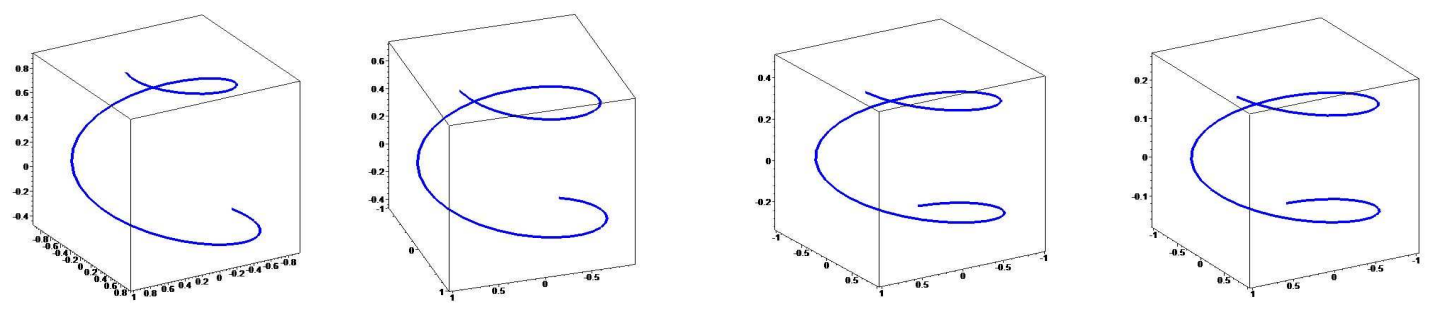

Figure 3.3: $T_{T}\left(T \wedge T_{T}\right)$-Smarandache Curve, $m=\frac{1}{3}, \frac{1}{5}, \frac{1}{8}, \frac{1}{16}$ and $s=[-5 . .5]$

Theorem 3.6. The geodesic curvature $K_{g}^{\alpha_{3}}$ according to $\alpha_{3}(s)$-Smarandache curve is given by

$$
K_{g}^{\alpha_{3}}=\frac{\tan ^{4}(n s)}{\left(1+2 \tan ^{2}(n s)\right)^{\frac{5}{2}}}\left(2 \chi_{4}-\tan (n s) \chi_{5}+\tan (n s) \chi_{6}\right),
$$

where the coefficients $\chi_{4}, \chi_{5}$ and $\chi_{6}$ are

$$
\begin{aligned}
& \chi_{4}=\frac{2}{\tan (n s)}\left(\frac{1}{\tan (n s)}\right)^{\prime}+\frac{1}{\tan (n s)}+\frac{2}{\tan ^{3}(n s)}, \\
& \chi_{5}=-1-\left(\frac{1}{\tan (n s)}\right)^{\prime}-\frac{3}{\tan ^{2}(n s)}-\frac{2}{\tan ^{4}(n s)}, \\
& \chi_{6}=-\frac{1}{\tan ^{2}(n s)}+\left(\frac{1}{\tan (n s)}\right)^{\prime}-\frac{2}{\tan ^{4}(n s)} .
\end{aligned}
$$

Proof. If we take the derivative of (3.7) and from the equation (2.3) we get

$$
\left(T_{T}\right)_{\alpha_{3}} \cdot \frac{d s^{*}}{d s}=\frac{1}{\sqrt{2}}\left(-T-\frac{1}{\tan (n s)} T_{T}+\frac{1}{\tan (n s)}\left(T \wedge T_{T}\right)\right)
$$

if take the norm of (3.8) we have, $\frac{d s^{*}}{d s}=\frac{\sqrt{\tan ^{2}(n s)+2}}{\tan (n s) \sqrt{2}}$. We obtain the tangent of $\alpha_{3}(s)$-Smarandahce curve as in

$$
\left(T_{T}\right)_{\alpha_{3}}=\frac{1}{\sqrt{\tan ^{2}(n s)+2}}\left(-\tan (n s) T-T_{T}+\left(T \wedge T_{T}\right)\right)
$$

The derivative of (3.9) is

$$
\left(T_{T}\right)_{\alpha_{3}}^{\prime}=\frac{\tan ^{4}(n s) \sqrt{2}}{\left(\tan ^{2}(n s)+2\right)^{2}}\left(\chi_{4} T+\chi_{5} T_{T}+\chi_{6}\left(T \wedge T_{T}\right)\right)
$$


From equations (3.7) and (3.9) we have

$$
\left(T \wedge T_{T}\right)_{\alpha_{3}}=\frac{1}{\sqrt{2\left(\tan ^{2}(n s)+2\right)}}\left(2 T-\tan (n s) T_{T}+\tan (n s)\left(T \wedge T_{T}\right)\right) .
$$

So the geodesic curvature from the equation $(2.3)$ is

$$
K_{g}^{\alpha_{3}}=\frac{\tan ^{4}(n s)}{\left(1+2 \tan ^{2}(n s)\right)^{\frac{5}{2}}}\left(2 \chi_{4}-\tan (n s) \chi_{5}+\tan (n s) \chi_{6}\right) .
$$

Definition 3.7. Let $\alpha=\alpha(s)$ be a curve and $\left\{T, T_{T}, T \wedge T_{T}\right\}$ be Sabban frame of this curve. Then $T T_{T}\left(T \wedge T_{T}\right)$-Smarandache curve is given by

$$
\alpha_{4}(s)=\frac{1}{\sqrt{3}}\left(T+T_{T}+\left(T \wedge T_{T}\right)\right)
$$

According to equation (2.4) we can parameterize the $\alpha_{1}(s)$-Smarandache curve as in the following form

$$
\begin{aligned}
\alpha_{4}(s)= & \frac{1}{\sqrt{3}}\left(\cos (s)(\cos (n s)-\sin (n s))+n \sin (s)(\cos (n s)+\sin (n s))+\frac{n}{m} \sin (s),\right. \\
& \left.\sin (s)(\cos (n s)-\sin (n s))-n \cos (s)(\cos (n s)+\sin (n s))-\frac{n}{m} \cos (s),-\frac{n}{m}(\cos (n s)+\sin (n s))+n\right) .
\end{aligned}
$$

The shape of this curve is given in Figure (3.4)
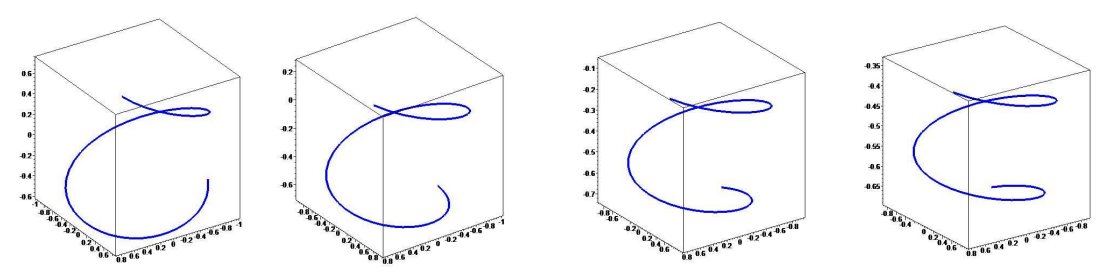

Figure 3.4: $T T_{T}\left(T \wedge T_{T}\right)$-Smarandache Curve, $m=\frac{1}{3}, \frac{1}{5}, \frac{1}{8}, \frac{1}{16}$ and $s=[-5,5]$

Theorem 3.8. The geodesic curvature $K_{g}^{\alpha_{4}}$ according to $\alpha_{4}(s)$-Smarandache curve is given as

$$
K_{g}^{\alpha_{4}}=\frac{\tan ^{4}(n s)\left((2-\tan (n s)) \chi_{7}-(1+\tan (n s)) \chi_{8}+(2 \tan (n s)-1) \chi_{9}\right)}{\left(4 \sqrt{2}\left(\tan ^{2}(n s)-\tan (n s)+1\right)^{2}\right)^{\frac{5}{2}}},
$$

where the coefficients $\chi_{6}, \chi_{7}$ and $\chi_{8}$ are

$$
\begin{aligned}
\chi_{7} & =-\left(\frac{1}{\tan (n s)}\right)^{\prime}+\frac{2}{\tan (n s)}\left(\frac{1}{\tan (n s)}\right)^{\prime}-2+\frac{4}{\tan (n s)}-\frac{4}{\tan ^{2}(n s)}+\frac{2}{\tan ^{3}(n s)}, \\
\chi_{8} & =-\left(\frac{1}{\tan (n s)}\right)^{\prime}-\frac{1}{\tan (n s)}\left(\frac{1}{\tan (n s)}\right)^{\prime}-2-\frac{4}{\tan ^{2}(n s)}+\frac{2}{\tan (n s)}+\frac{2}{\tan ^{3}(n s)}-\frac{2}{\tan ^{4}(n s)}, \\
\chi_{9} & =\frac{1}{\tan (n s)}\left(\frac{1}{\tan (n s)}\right)^{\prime}+\frac{2}{\tan (n s)}-\frac{4}{\tan ^{2}(n s)}+\left(\frac{2}{\tan (n s)}\right)^{\prime}+\frac{4}{\tan ^{3}(n s)}-\frac{2}{\tan ^{4}(n s)} .
\end{aligned}
$$

Proof. If we take the derivative of (3.10) and from the equation (2.3) we get,

$$
\left(T_{T}\right)_{\alpha_{4}} \cdot \frac{d s^{*}}{d s}=\frac{1}{\sqrt{3}}\left(-T+\left(1-\frac{1}{\tan (n s)}\right) T_{T}+\frac{1}{\tan (n s)}\left(T \wedge T_{T}\right)\right),
$$

if we take the norm of (3.11) we have,

$$
\frac{d s^{*}}{d s}=\frac{\sqrt{2\left(\tan ^{2}(n s)-\tan (n s)+1\right)}}{\tan (n s) \sqrt{3}} .
$$

We obtain the tangent of $\alpha_{4}(s)$-Smarandahce curve as in

$$
\left(T_{T}\right)_{\alpha_{4}}=\frac{\left(-\tan (n s) T+(\tan (n s)-1) T_{T}+\left(T \wedge T_{T}\right)\right)}{\sqrt{2\left(\tan ^{2}(n s)-\tan (n s)+1\right)}} .
$$

The derivative of (3.12) is

$$
\left(T_{T}\right)_{\alpha_{4}}^{\prime}=\frac{\tan ^{2}(n s) \sqrt{3}\left(\chi_{7} T+\chi_{8} T_{T}+\chi_{9}\left(T \wedge T_{T}\right)\right)}{4\left(\tan ^{2}(n s)-\tan (n s)+1\right)^{2}}
$$


From equations (3.10) and (3.12) we have

$$
\left(T \wedge T_{T}\right)_{\alpha_{4}}=\frac{(-\tan (n s)+2) T-(\tan (n s)+1) T_{T}+(2 \tan (n s)-1)\left(T \wedge T_{T}\right)}{\sqrt{6\left(\tan ^{2}(n s)-\tan (n s)+1\right)}} .
$$

So the geodesic curvature from the equation $(2.3)$ is

$$
K_{g}^{\alpha_{4}}=\frac{\tan ^{4}(n s)\left((2-\tan (n s)) \chi_{7}-(1+\tan (n s)) \chi_{8}+(2 \tan (n s)-1) \chi_{9}\right)}{\left(4 \sqrt{2}\left(\tan ^{2}(n s)-\tan (n s)+1\right)^{2}\right)^{\frac{5}{2}}} .
$$

Definition 3.9. Let $\delta=\delta(s)$ be a curve and $\left\{N, T_{N}, N \wedge T_{N}\right\}$ be Sabban frame of this curve. Then $N T_{N}$-Smarandache curve is given by

$$
\delta_{1}(s)=\frac{1}{\sqrt{2}}\left(N+T_{N}\right)
$$

According to equation (2.6) we can parameterize the $\delta_{1}(s)$-Smarandache curve as in the following form

$$
\begin{aligned}
\delta_{1}(s)= & \frac{1}{\sqrt{2}}\left(-\frac{\tan (n s)}{\sqrt{\tan ^{2}(n s)+1}}(-\cos (s) \sin (n s)+n \sin (s) \cos (n s))+\frac{n \sin (s)}{m}-\cos (s) \cos (n s)-n \sin (s) \sin (n s),\right. \\
& -\sin (s) \cos (n s)+n \cos (s) \sin (n s)-\frac{n \cos (s)}{m}-\frac{\tan (n s)}{\sqrt{\tan ^{2}(n s)+1}}(-\sin (s) \sin (n s)-n \cos (s) \cos (n s)), \\
& \left.\frac{n \tan (n s)}{m \sqrt{\tan ^{2}(n s)+1}} \cos (n s)+n\right) .
\end{aligned}
$$

The shape of this curve is given in Figure (3.5)
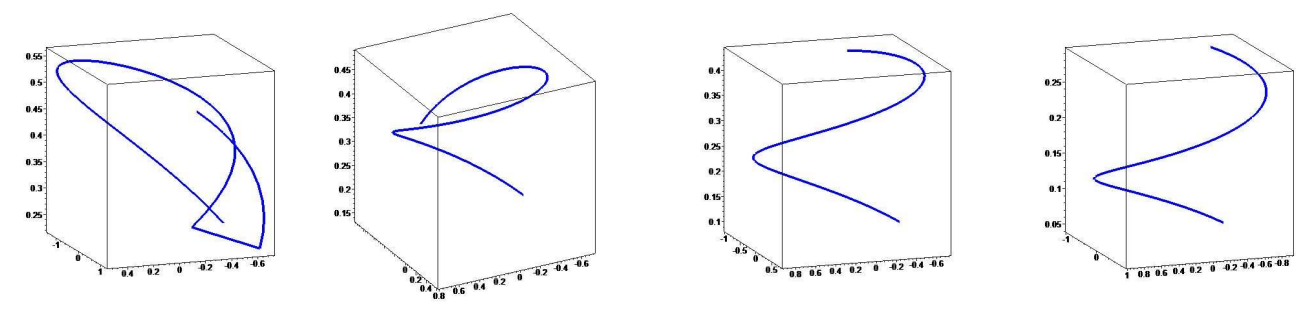

Figure 3.5: $N T_{N}$-Smarandache Curves , $m=\frac{1}{3}, \frac{1}{5}, \frac{1}{8}, \frac{1}{16}$ and $s=[-5,5]$

Theorem 3.10. The geodesic curvature $K_{g}^{\delta_{1}}$ according to $\delta_{1}(s)$-Smarandache curve is given by

$$
K_{g}^{\delta_{1}}=\frac{\left(1+\tan ^{2}(n s)\right)\left(-\tan (n s)^{\prime} \chi_{10}+\tan (n s)^{\prime} \chi_{11}+2 \sqrt{\tan ^{2}(n s)+1} \chi_{12}\right)}{\left(2 \sqrt{1+\tan ^{2}(n s)}-\left(\tan (n s)^{\prime}\right)^{2}\right)^{\frac{5}{2}}},
$$

where the coefficients $\chi_{10}, \chi_{11}$ and $\chi_{12}$ are

$$
\begin{aligned}
& \chi_{10}=-2-\left(\frac{-\tan (n s)^{\prime}}{\sqrt{\tan ^{2}(n s)+1}}\right)^{2}+\frac{-\tan (n s)^{\prime}}{\sqrt{\tan ^{2}(n s)+1}}\left(\frac{-\tan (n s)^{\prime}}{\sqrt{\tan ^{2}(n s)+1}}\right)^{\prime}, \\
& \chi_{11}=-2-\frac{-\tan (n s)^{\prime}}{\sqrt{\tan ^{2}(n s)+1}}\left(\frac{-\tan (n s)^{\prime}}{\sqrt{\tan ^{2}(n s)+1}}\right)^{\prime}-3\left(\frac{-\tan (n s)^{\prime}}{\sqrt{\tan ^{2}(n s)+1}}\right)^{2}-\left(\frac{-\tan (n s)^{\prime}}{\sqrt{\tan ^{2}(n s)+1}}\right)^{4}, \\
& \chi_{12}=\frac{-2 \tan (n s)^{\prime}}{\sqrt{\tan ^{2}(n s)+1}}+2\left(\frac{-\tan (n s)^{\prime}}{\sqrt{\tan ^{2}(n s)+1}}\right)^{\prime}+\left(\frac{-\tan (n s)^{\prime}}{\sqrt{\tan ^{2}(n s)+1}}\right)^{3} .
\end{aligned}
$$

Proof. If we take the derivative of equation (3.13) and from the equation (2.5) we have

$$
\left(T_{N}\right)_{\delta_{1}} \cdot \frac{d s^{*}}{d s}=\frac{1}{\sqrt{2}}\left(-N+T_{N}+\frac{-\tan (n s)^{\prime}}{\sqrt{\tan ^{2}(n s)+1}}\left(N \wedge T_{N}\right)\right)
$$

if we take the norm of equation (3.13) we get

$$
\frac{d s^{*}}{d s}=\frac{\sqrt{2\left(1+\tan ^{2}(n s)\right)-\left(\tan (n s)^{\prime}\right)^{2}}}{\sqrt{2} \sqrt{1+\tan ^{2}(n s)}} .
$$


We obtain the tangent of $\delta_{1}(s)$-Smarandahce curve as in

$$
\left(T_{N}\right)_{\delta_{1}}=\frac{-\sqrt{\tan ^{2}(n s)+1} N+\sqrt{\tan ^{2}(n s)+1} T_{N}-\tan (n s)^{\prime}\left(N \wedge T_{N}\right)}{\sqrt{2\left(1+\tan ^{2}(n s)\right)-\left(\tan (n s)^{\prime}\right)^{2}}} .
$$

The derivative of (3.13) is

$$
\left(T_{N}\right)_{\delta_{1}}^{\prime}=\frac{\left(\tan ^{2}(n s)+1\right) \sqrt{2}\left(\chi_{10} N+\chi_{11} T_{N}+\chi_{12}\left(N \wedge T_{N}\right)\right)}{\left(2\left(\tan ^{2}(n s)+1\right)-\left(\tan (n s)^{\prime}\right)^{2}\right)^{2}} .
$$

From equations (3.13) and (3.14) we have

$$
\left(N \wedge T_{N}\right)_{\delta_{1}}=\frac{\left(1+\tan ^{2}(n s)\right)^{4}\left(-\tan (n s)^{\prime}\left(N-T_{N}\right)+2 \sqrt{1+\tan ^{2}(n s)}\left(N \wedge T_{N}\right)\right)}{\sqrt{2\left(2\left(1+\tan ^{2}(n s)\right)-\left(\tan (n s)^{\prime}\right)^{2}\right)}} .
$$

So the geodesic curvature from the equation (2.5) is

$$
K_{g}^{\delta_{1}}=\frac{\left(1+\tan ^{2}(n s)\right)\left(-\tan (n s)^{\prime} \chi_{10}+\tan (n s)^{\prime} \chi_{11}+2 \sqrt{\tan ^{2}(n s)+1} \chi_{12}\right)}{\left(2 \sqrt{1+\tan ^{2}(n s)}-\left(\tan (n s)^{\prime}\right)^{2}\right)^{\frac{5}{2}}} .
$$

Definition 3.11. Let $\delta=\delta(s)$ be a curve and $\left\{N, T_{N}, N \wedge T_{N}\right\}$ be Sabban frame of this curve. Then $N\left(N \wedge T_{N}\right)$-Smarandache curve is given by

$$
\delta_{2}(s)=\frac{1}{\sqrt{2}}\left(N+\left(N \wedge T_{N}\right)\right) .
$$

According to equation (2.6) we can parameterize the $\delta_{2}(s)$-Smarandache curve as in the following form

$$
\begin{aligned}
\delta_{2}(s)= & \frac{1}{\sqrt{2}}\left(\frac{\tan (n s)}{\sqrt{\tan ^{2}(n s)+1}}(-\cos (s) \cos (n s)-n \sin (s) \sin (n s))-\cos (s) \sin (n s)+n \sin (s) \cos (n s)+\frac{n \sin (s)}{m}\right. \\
& +\frac{\tan (n s)}{\sqrt{\tan ^{2}(n s)+1}}(-\sin (s) \cos (n s)+n \cos (s) \sin (n s))-\sin (s) \sin (n s)-n \cos (s) \cos (n s)-\frac{n \cos (s)}{m} \\
& \left.\frac{n \tan (n s)}{m \sqrt{\tan ^{2}(n s)+1}} \sin (n s)-\frac{n}{m} \cos (n s)+n\right) .
\end{aligned}
$$

The shape of this curve is given in Figure (3.6)
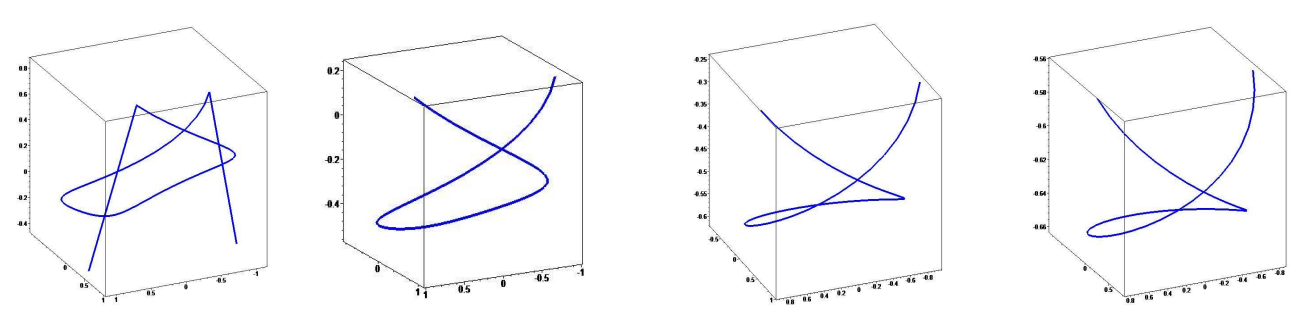

Figure 3.6: $N\left(N \wedge T_{N}\right)$-Smarandache Curve, $m=\frac{1}{3}, \frac{1}{5}, \frac{1}{8}, \frac{1}{16}$ and $s=[-5,5]$

Theorem 3.12. The geodesic curvature $K_{g}^{\delta_{2}}$ according to $\delta_{2}(s)$-Smarandache curve is given by

$$
K_{g}^{\delta_{2}}=\frac{\sqrt{\tan (n s)^{2}+1}-\tan (n s)^{\prime}}{\sqrt{\tan (n s)^{2}+1}} .
$$

Proof. If we take the derivative of equation (3.15) and from the equation (2.5) we get

$$
\left(T_{N}\right)_{\delta_{2}} \cdot \frac{d s^{*}}{d s}=\frac{1}{\sqrt{2}}\left(T_{N}-\frac{-\tan (n s)^{\prime}}{\sqrt{\tan ^{2}(n s)+1}} T_{N}\right)
$$

if we take the norm of equation (3.16) we have

$$
\frac{d s^{*}}{d s}=\frac{\sqrt{\tan (n s)^{2}+1}+\tan (n s)^{\prime}}{\sqrt{2} \sqrt{\tan (n s)^{2}+1}}
$$


We obtain the tangent of $\delta_{2}(s)$-Smarandahce curve as in

$$
\left(T_{N}\right)_{\delta_{2}}=T_{N}
$$

The derivative of (3.17) is

$$
\left(T_{N}\right)_{\delta_{2}}^{\prime}=\frac{\sqrt{2}\left(-\sqrt{\tan ^{2}(n s)+1} N-\tan (n s)^{\prime}\left(N \wedge T_{N}\right)\right)}{\sqrt{\tan (n s)^{2}+1}+\tan (n s)^{\prime}} .
$$

From equations (3.15) and (3.17) we have

$$
\left(N \wedge T_{N}\right)_{\delta_{2}}=\frac{1}{\sqrt{2}}\left(-N+\left(N \wedge T_{N}\right)\right) .
$$

So the geodesic curvature from the equation (2.5) is

$$
K_{g}^{\delta_{2}}=\frac{\sqrt{\tan (n s)^{2}+1}-\tan (n s)^{\prime}}{\sqrt{\tan (n s)^{2}+1}} .
$$

Definition 3.13. Let $\delta=\delta(s)$ be a curve and $\left\{N, T_{N}, N \wedge T_{N}\right\}$ be Sabban frame of this curve. Then $T_{N}\left(N \wedge T_{N}\right)$-Smarandache curve $\left(\delta_{3}(s)\right.$-Smarandache curve) is given by

$$
\delta_{3}(s)=\frac{1}{\sqrt{2}}\left(T_{N}+\left(N \wedge T_{N}\right)\right)
$$

According to equation (2.6) we can parameterize the $\delta_{3}(s)$-Smarandache curve as in the following form

$$
\begin{aligned}
\delta_{3}(s)= & \frac{1}{\sqrt{2}}\left(-\cos (s) \cos (n s)-n \sin (s) \sin (n s)-\cos (s) \sin (n s)+\frac{\tan (n s)}{\sqrt{\tan ^{2}(n s)+1}}(-\cos (s) \cos (n s)-n \sin (s) \sin (n s))\right. \\
& -\frac{\tan (n s)}{\sqrt{\tan ^{2}(n s)+1}}(-\cos (s) \sin (n s)+n \sin (s) \cos (n s))+n \sin (s) \cos (n s),-\sin (s) \sin (n s)-n \cos (s) \cos (n s) \\
& -\sin (s) \cos (n s)+n \cos (s) \sin (n s)+\frac{\tan (n s)}{\sqrt{\tan ^{2}(n s)+1}}(-\sin (s) \cos (n s)+n \cos (s) \sin (n s)) \\
& \left.-\frac{\tan (n s)}{\sqrt{\tan ^{2}(n s)+1}}(-\sin (s) \sin (n s)-n \cos (s) \cos (n s)), \frac{n \tan (n s)}{m \sqrt{\tan ^{2}(n s)+1}}(\cos (n s)+\sin (n s))-\frac{n}{m} \cos (n s)\right)
\end{aligned}
$$

The shape of this curve is given in Figure (3.7)
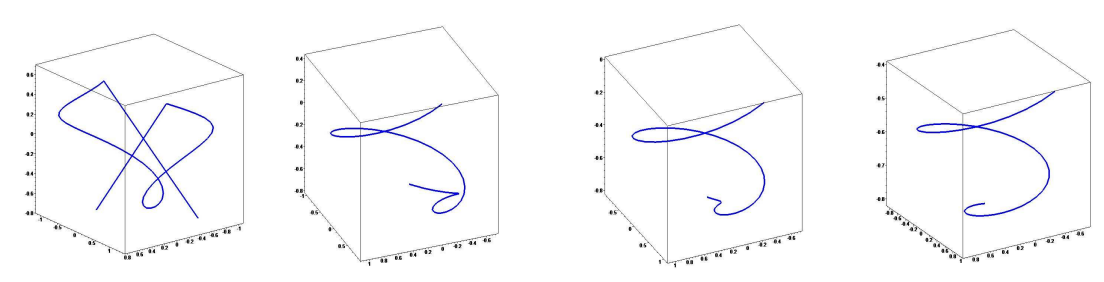

Figure 3.7: $T_{N}(N \wedge T N)$-Smarandache Curve, $m=\frac{1}{3}, \frac{1}{5}, \frac{1}{8}, \frac{1}{16}$ and $s=[-5,5]$

Theorem 3.14. The geodesic curvature $K_{g}^{\delta_{3}}$ according to $\delta_{3}(s)$-Smarandache curve is given by

$$
K_{g}^{\delta_{3}}=\frac{\left(\tan ^{2}(n s)+1\right)^{4}\left(\left(-2 \tan (n s)^{\prime}\right) \chi_{13}-\sqrt{\tan (n s)^{2}+1}\left(\chi_{14}-\chi_{15}\right)\right)}{\left(1+\tan ^{2}(n s)+\left(-\tan (n s)^{\prime}\right)^{2}\right)^{\frac{5}{2}}},
$$

where the coefficients $\chi_{13}, \chi_{14}$ and $\chi_{15}$ are

$$
\begin{aligned}
& \chi_{13}=2 \frac{-\tan (n s)^{\prime}}{\sqrt{\tan ^{2}(n s)+1}}\left(\frac{-\tan (n s)^{\prime}}{\sqrt{\tan ^{2}(n s)+1}}\right)^{\prime}+\frac{-\tan (n s)^{\prime}}{\sqrt{\tan ^{2}(n s)+1}}+2\left(\frac{-\tan (n s)^{\prime}}{\sqrt{\tan ^{2}(n s)+1}}\right)^{3}, \\
& \chi_{14}=-1-\left(\frac{-\tan (n s)^{\prime}}{\sqrt{\tan ^{2}(n s)+1}}\right)^{\prime}-\left(\frac{-3 \tan (n s)^{\prime}}{\sqrt{\tan ^{2}(n s)+1}}\right)^{2}-\left(\frac{-2 \tan (n s)^{\prime}}{\sqrt{\tan ^{2}(n s)+1}}\right)^{4}, \\
& \chi_{15}=-\left(\frac{-\tan (n s)^{\prime}}{\sqrt{\tan ^{2}(n s)+1}}\right)^{2}+\left(\frac{-2 \tan (n s)^{\prime}}{\sqrt{\tan ^{2}(n s)+1}}\right)^{\prime}-\left(\frac{-2 \tan (n s)^{\prime}}{\sqrt{\tan ^{2}(n s)+1}}\right)^{4} .
\end{aligned}
$$


Proof. If we take the derivative of equation (3.18) and from the equation (2.5) we get

$$
\left(T_{N}\right)_{\delta_{3}} \cdot \frac{d s^{*}}{d s}=\frac{1}{\sqrt{2}}\left(-N-\frac{-\tan (n s)^{\prime}}{\sqrt{\tan ^{2}(n s)+1}} T_{N}+\frac{-\tan (n s)^{\prime}}{\sqrt{\tan ^{2}(n s)+1}}\left(N \wedge T_{N}\right)\right),
$$

if we take the norm of equation (3.19) we have

$$
\frac{d s^{*}}{d s}=\frac{\sqrt{1+\tan ^{2}(n s)+2\left(-\tan (n s)^{\prime}\right)^{2}}}{\sqrt{2} \sqrt{\tan ^{2}(n s)+1}} .
$$

We obtain the tangent of $\delta_{3}(s)$-Smarandahce curve as in

$$
\left(T_{N}\right)_{\delta_{3}}=\frac{-\sqrt{\tan ^{2}(n s)+1} N+\tan (n s)^{\prime} T_{N}-\tan (n s)^{\prime}\left(N \wedge T_{N}\right)}{\sqrt{1+\tan ^{2}(n s)+2\left(-\tan (n s)^{\prime}\right)^{2}}}
$$

The derivative of (3.20) is

$$
\left(T_{N}\right)_{\delta_{3}}^{\prime}=\frac{\sqrt{2}\left(\tan ^{2}(n s)+1\right)^{2}}{\left(1+\tan ^{2}(n s)+2\left(-\tan (n s)^{\prime}\right)^{2}\right)^{2}}\left(\chi_{13} N+\chi_{14} T_{N}+\chi_{15}\left(N \wedge T_{N}\right)\right) .
$$

From equations (3.18) and (3.20) we have

$$
\left(N \wedge T_{N}\right)_{\delta_{3}}=\frac{\left(-2 \tan (n s)^{\prime} N-\sqrt{1+\tan ^{2}(n s)} T_{N}+\sqrt{1+\tan ^{2}(n s)}\left(N \wedge T_{N}\right)\right)}{\sqrt{2\left(1+\tan ^{2}(n s)+2\left(-\tan (n s)^{\prime}\right)^{2}\right)}} .
$$

So the geodesic curvature from the equation (2.5) is

$$
K_{g}^{\delta_{3}}=\frac{\left(\tan ^{2}(n s)+1\right)^{4}\left(\left(-2 \tan (n s)^{\prime}\right) \chi_{13}-\sqrt{\tan (n s)^{2}+1}\left(\chi_{14}-\chi_{15}\right)\right)}{\left(1+\tan ^{2}(n s)+\left(-\tan (n s)^{\prime}\right)^{2}\right)^{\frac{5}{2}}} .
$$

Definition 3.15. Let $\delta=\delta(s)$ be a curve and $\left\{N, T_{N}, N \wedge T_{N}\right\}$ be Sabban frame of this curve. Then $N T_{N}\left(N \wedge T_{N}\right)$-Smarandache curve $\left(\delta_{4}(s)\right.$-Smarandache curve) is given by

$$
\delta_{4}(s)=\frac{1}{\sqrt{3}}\left(N+T_{N}+\left(N \wedge T_{N}\right)\right)
$$

According to equation (2.6) we can parameterize the $\delta_{4}(s)$-Smarandache curve as in the following form

$$
\begin{aligned}
\delta_{4}(s)= & \frac{1}{\sqrt{3}}\left(-\cos (s) \cos (n s)-n \sin (s) \sin (n s)-\cos (s) \sin (n s)+\frac{\tan (n s)}{\sqrt{\tan ^{2}(n s)+1}}(-\cos (s) \cos (n s)-n \sin (s) \sin (n s))+\frac{n \sin (s)}{m}\right. \\
& -\frac{\tan (n s)}{\sqrt{\tan ^{2}(n s)+1}}(-\cos (s) \sin (n s)+n \sin (s) \cos (n s))+n \sin (s) \cos (n s),-\sin (s) \sin (n s) \\
& +\frac{\tan (n s)}{\sqrt{\tan ^{2}(n s)+1}}(-\sin (s) \cos (n s)+n \cos (s) \sin (n s))-n \cos (s) \cos (n s)-\sin (s) \cos (n s)+n \cos (s) \sin (n s) \\
& \left.-\frac{\tan (n s)}{\sqrt{\tan ^{2}(n s)+1}}(-\sin (s) \sin (n s)-n \cos (s) \cos (n s))-\frac{n \cos (s)}{m}, \frac{n \tan (n s)}{m \sqrt{\tan ^{2}(n s)+1}}(\cos (n s)+\sin (n s))-\frac{n}{m} \cos (n s)+n\right) .
\end{aligned}
$$

The shape of this curve is given in Figure (3.8)
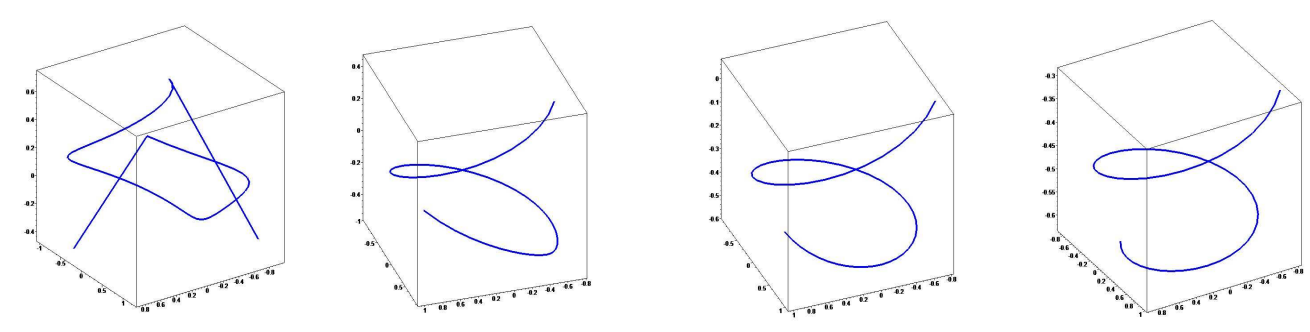

Figure 3.8: $T T_{N}\left(T \wedge T_{N}\right)$-Smarandache Curve, $m=\frac{1}{3}, \frac{1}{5}, \frac{1}{8}, \frac{1}{16}$ and $s=[-5,5]$ 
Theorem 3.16. The geodesic curvature $K_{g}^{\delta_{4}}$ according to $\delta_{4}(s)$-Smarandache curve is given by

$$
\begin{aligned}
K_{g}^{\delta_{4}=} & \frac{\left(\left(-2 \tan (n s)^{\prime}-\sqrt{\tan (n s)^{2}+1}\right) \chi_{16}-\chi_{17}\left(\sqrt{\tan (n s)^{2}+1}-\tan (n s)^{\prime}\right)\right)}{\left(4 \sqrt{2}\left(1+\tan ^{2}(n s)+\sqrt{1+\tan ^{2}(n s)} \tan (n s)^{\prime}+\left(-\tan (n s)^{\prime}\right)^{2}\right)^{2}\right)^{\frac{5}{2}}} \\
& +\frac{\left(2 \sqrt{\tan (n s)^{2}+1}+\tan (n s)^{\prime}\right) \chi_{18}}{\left(4 \sqrt{2}\left(1+\tan ^{2}(n s)+\sqrt{1+\tan ^{2}(n s)} \tan (n s)^{\prime}+\left(-\tan (n s)^{\prime}\right)^{2}\right)^{2}\right)^{\frac{5}{2}}},
\end{aligned}
$$

where the coefficients $\chi_{16}, \chi_{17}$ and $\chi_{18}$ are

$$
\begin{aligned}
\chi_{16}= & \left(\frac{\tan (n s)^{\prime}}{\sqrt{\tan ^{2}(n s)+1}}\right)^{\prime}+\frac{-2 \tan (n s)^{\prime}}{\sqrt{\tan ^{2}(n s)+1}}\left(\frac{-\tan (n s)^{\prime}}{\sqrt{\tan ^{2}(n s)+1}}\right)^{\prime}-2+\frac{-4 \tan (n s)^{\prime}}{\sqrt{\tan ^{2}(n s)+1}}+\left(\frac{4 \tan (n s)^{\prime}}{\sqrt{\tan ^{2}(n s)+1}}\right)^{2}+\left(\frac{-2 \tan (n s)^{\prime}}{\sqrt{\tan ^{2}(n s)+1}}\right)^{3} \\
\chi_{17}= & -\left(\frac{-\tan (n s)^{\prime}}{\sqrt{\tan ^{2}(n s)+1}}\right)^{\prime}-\frac{-\tan (n s)^{\prime}}{\sqrt{\tan ^{2}(n s)+1}}\left(\frac{-\tan (n s)^{\prime}}{\sqrt{\tan ^{2}(n s)+1}}\right)^{\prime}-2+\left(\frac{4 \tan (n s)^{\prime}}{\sqrt{\tan ^{2}(n s)+1}}\right)^{2}-\frac{2 \tan (n s)^{\prime}}{\sqrt{\tan ^{2}(n s)+1}}-\left(\frac{2 \tan (n s)^{\prime}}{\sqrt{\tan ^{2}(n s)+1}}\right)^{3} \\
& +\left(\frac{2 \tan (n s)^{\prime}}{\sqrt{\tan ^{2}(n s)+1}}\right)^{4}, \\
\chi_{18}= & \frac{-\tan (n s)^{\prime}}{\sqrt{\tan ^{2}(n s)+1}}\left(\frac{-\tan (n s)^{\prime}}{\sqrt{\tan ^{2}(n s)+1}}\right)^{\prime}+\frac{-2 \tan (n s)^{\prime}}{\sqrt{\tan ^{2}(n s)+1}}-4\left(\frac{-\tan (n s)^{\prime}}{\sqrt{\tan ^{2}(n s)+1}}\right)^{2}+2\left(\frac{-\tan (n s)^{\prime}}{\sqrt{\tan ^{2}(n s)+1}}\right)^{\prime}+4\left(\frac{-\tan (n s)^{\prime}}{\sqrt{\tan ^{2}(n s)+1}}\right)^{3} \\
& -2\left(\frac{-\tan (n s)^{\prime}}{\sqrt{\tan ^{2}(n s)+1}}\right)^{4} .
\end{aligned}
$$

Proof. If we take the derivative of equation (3.21) and from the equation (2.5) we have

$$
\left(T_{N}\right)_{\delta_{4}} \cdot \frac{d s^{*}}{d s}=\frac{1}{\sqrt{3}}\left(-N+\left(1-\frac{-\tan (n s)^{\prime}}{\sqrt{\tan ^{2}(n s)+1}}\right) T_{N}+\frac{-\tan (n s)^{\prime}}{\sqrt{\tan ^{2}(n s)+1}}\left(N \wedge T_{N}\right)\right)
$$

if we take the norm of equation (3.22) we get

$$
\frac{d s^{*}}{d s}=\frac{\sqrt{2\left(1+\tan ^{2}(n s)+\tan (n s)^{\prime} \sqrt{\tan ^{2}(n s)+1}+\left(-\tan (n s)^{\prime}\right)^{2}\right)}}{\sqrt{3} \sqrt{\tan ^{2}(n s)+1}} .
$$

We obtain the tangent of $\delta_{4}(s)$-Smarandahce curve as in

$$
\left(T_{N}\right)_{\delta_{4}}=\frac{-\sqrt{\tan ^{2}(n s)+1} N+\left(\sqrt{\tan ^{2}(n s)+1}+\tan (n s)^{\prime}\right) T_{N}-\tan (n s)^{\prime}\left(N \wedge T_{N}\right)}{\sqrt{2\left(1+\tan ^{2}(n s)+\tan (n s)^{\prime} \sqrt{\tan ^{2}(n s)+1}+\left(-\tan (n s)^{\prime}\right)^{2}\right)}} .
$$

The derivative of (3.23) is

$$
\left(T_{N}\right)_{\delta_{4}}^{\prime}=\frac{\sqrt{3}\left(\chi_{16} N+\chi_{17} T_{N}+\chi_{18}\left(N \wedge T_{N}\right)\right)}{4\left(1+\tan ^{2}(n s)+\tan (n s)^{\prime} \sqrt{\tan ^{2}(n s)+1}+\left(-\tan (n s)^{\prime}\right)^{2}\right)^{2}} .
$$

From equations (3.21) and (3.23) we have

$$
\begin{aligned}
\left(N \wedge T_{N}\right)_{\delta_{4}}= & \frac{\left(-\left(\sqrt{\tan ^{2}(n s)+1}+2 \tan (n s)^{\prime}\right) N-\left(\sqrt{\tan ^{2}(n s)+1}-\tan (n s)^{\prime}\right) T_{N}\right)}{\sqrt{6\left(1+\tan ^{2}(n s)+\tan (n s)^{\prime} \sqrt{\tan ^{2}(n s)+1}+\left(-\tan (n s)^{\prime}\right)^{2}\right.}} \\
& +\frac{\left(2 \sqrt{\tan ^{2}(n s)+1}+\tan (n s)^{\prime}\right)\left(N \wedge T_{N}\right)}{\sqrt{6\left(1+\tan ^{2}(n s)+\tan (n s)^{\prime} \sqrt{\tan ^{2}(n s)+1}+\left(-\tan (n s)^{\prime}\right)^{2}\right)}}
\end{aligned}
$$

So the geodesic curvature from the equation (2.5) is

$$
\begin{aligned}
K_{g}^{\delta_{4}=} & \frac{\left(\left(-2 \tan (n s)^{\prime}-\sqrt{\tan (n s)^{2}+1}\right) \chi_{16}-\chi_{17}\left(\sqrt{\tan (n s)^{2}+1}-\tan (n s)^{\prime}\right)\right)}{\left(4 \sqrt{2}\left(1+\tan ^{2}(n s)+\sqrt{1+\tan ^{2}(n s)} \tan (n s)^{\prime}+\left(-\tan (n s)^{\prime}\right)^{2}\right)^{2}\right)^{\frac{5}{2}}} \\
& +\frac{\left(2 \sqrt{\tan (n s)^{2}+1}+\tan (n s)^{\prime}\right) \chi_{18}}{\left(4 \sqrt{2}\left(1+\tan ^{2}(n s)+\sqrt{1+\tan ^{2}(n s)} \tan (n s)^{\prime}+\left(-\tan (n s)^{\prime}\right)^{2}\right)^{2}\right)^{\frac{5}{2}}} .
\end{aligned}
$$


Definition 3.17. Let $\zeta=\zeta(s)$ be a curve and $\left\{B, T_{B}, B \wedge T_{B}\right\}$ be Sabban frame of this curve. Then $B T_{B}$-Smarandache curve $\left(\zeta_{1}(s)\right.$ Smarandache curve) is given by

$$
\zeta_{1}(s)=\frac{1}{\sqrt{2}}\left(B+T_{B}\right)
$$

According to equation (2.8) we can parameterize the $\zeta_{1}(s)$-Smarandache curve as in the following form

$$
\zeta_{1}(s)=\frac{1}{\sqrt{2}}\left(-\cos (s) \cos (n s)-n \sin (s) \sin (n s)+\frac{n}{m} \sin (s),-\sin (s) \cos (n s)-n \cos (s) \sin (n s)-\frac{n}{m} \cos (s), \frac{n}{m} \sin (n s)+n\right) .
$$

The shape of this curve is given in Figure (3.9)
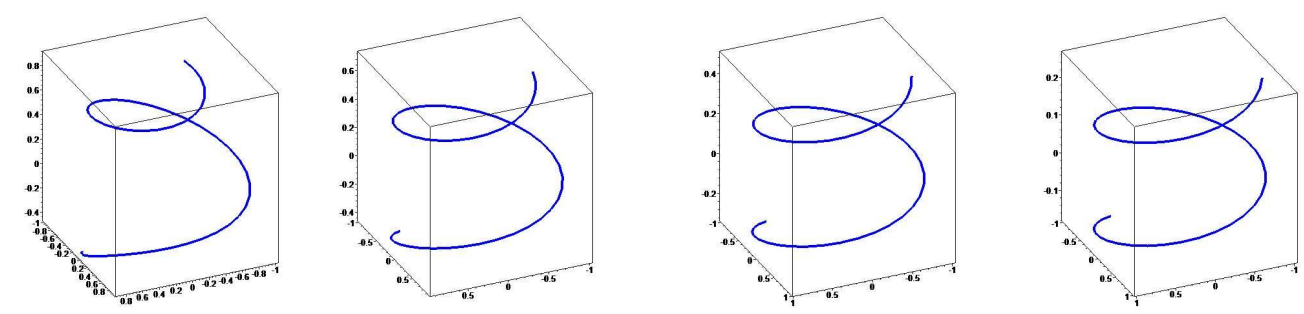

Figure 3.9: $B T_{B}\left(B \wedge T_{B}\right)$-Smarandache Curve, $m=\frac{1}{3}, \frac{1}{5}, \frac{1}{8}, \frac{1}{16}$ and $s=[-5,5]$

Theorem 3.18. The geodesic curvature $K_{g}^{\zeta_{1}}$ according to $\zeta_{1}(s)$-Smarandache curve is

$$
K_{g}^{\zeta_{1}}=\frac{1}{\left(2+(\tan (n s))^{2}\right)^{\frac{5}{2}}}\left(\chi_{19} \tan (n s)-\chi_{20} \tan (n s)+2 \chi_{21}\right),
$$

where the coefficients $\chi_{19}, \chi_{20}$ and $\chi_{21}$ are

$$
\begin{aligned}
& \chi_{19}=-2-\tan ^{2}(n s)+\tan (n s) \tan (n s)^{\prime}, \\
& \chi_{20}=-2-\tan (n s) \tan (n s)^{\prime}-3 \tan ^{2}(n s)-\tan ^{4}(n s), \\
& \chi_{21}=2 \tan (n s)+2 \tan (n s)^{\prime}+\tan ^{3}(n s) .
\end{aligned}
$$

Proof. If we take the derivative of equation (3.24) and from the equation (2.7) we get

$$
\left(T_{B}\right)_{\zeta_{1}} \cdot \frac{d s^{*}}{d s}=\frac{1}{\sqrt{2}}\left(-B+T_{B}+\tan (n s)\left(B \wedge T_{B}\right)\right)
$$

if we take the norm of equation (3.25) we have

$$
\frac{d s^{*}}{d s}=\frac{1}{\sqrt{2}} \sqrt{2+\tan ^{2}(n s)} .
$$

We obtain the tangent of $\zeta_{1}(s)$-Smarandahce curve as in

$$
\left(T_{B}\right)_{\zeta_{1}}=\frac{1}{\sqrt{2+\tan ^{2}(n s)}}\left(-B+T_{N}+\tan (n s)\left(B \wedge T_{B}\right)\right) .
$$

The derivative of (3.26) is

$$
\left(T_{B}\right)_{\zeta_{1}}^{\prime} \cdot \frac{d s^{*}}{d s}=\frac{\sqrt{2}}{\left(2+\tan ^{2}(n s)\right)^{2}}\left(\chi_{19} B+\chi_{20} T_{B}+\chi_{21}\left(B \wedge T_{B}\right)\right) .
$$

From equations (3.24) and (3.26) we have

$$
\left(B \wedge T_{B}\right)_{\zeta_{1}}=\frac{1}{\sqrt{4+2 \tan ^{2}(n s)}}\left(\tan (n s) N-\tan (n s) T_{B}+2\left(B \wedge T_{B}\right)\right) .
$$

So the geodesic curvature from the equation (2.7) is

$$
K_{g}^{\zeta_{1}}=\frac{1}{\left(2+(\tan (n s))^{2}\right)^{\frac{5}{2}}}\left(\chi_{19} \tan (n s)-\chi_{20} \tan (n s)+2 \chi_{21}\right) .
$$


Definition 3.19. Let $\zeta=\zeta(s)$ be a curve and $\left\{B, T_{B}, B \wedge T_{B}\right\}$ be Sabban frame of this curve. Then $B\left(B \wedge T_{B}\right)$-Smarandache curve $\left(\zeta_{2}(s)\right.$ Smarandache curve) is given by

$$
\zeta_{2}(s)=\frac{1}{\sqrt{2}}\left(B+\left(B \wedge T_{B}\right)\right)
$$

According to equation (2.8) we can parameterize the $\zeta_{2}(s)$-Smarandache curve as in the following form

$$
\begin{aligned}
\zeta_{2}(s)= & \frac{1}{\sqrt{2}}(-\cos (s)(\cos (n s)-\sin (n s))-n \sin (s)(\cos (n s)+\sin (n s)),-\sin (s)(\cos (n s)-\sin (n s))+n \cos (s)(\cos (n s)+\sin (n s)), \\
& \left.\frac{n}{m}(\cos (n s)+\sin (n s))\right) .
\end{aligned}
$$

The shape of this curve is given in Figure (3.10)
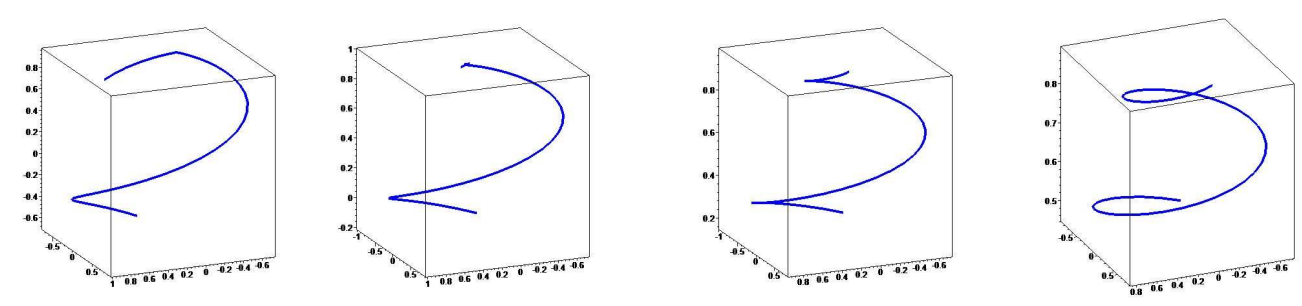

Figure 3.10: $B\left(B \wedge T_{B}\right)$-Smarandache Curve, $m=\frac{1}{3}, \frac{1}{5}, \frac{1}{8}, \frac{1}{16}$ and $s=[-5,5]$

Theorem 3.20. The geodesic curvature $K_{g}^{\zeta_{2}}$ according to $\zeta_{2}(s)$-Smarandache curve is

$$
K_{g}^{\zeta_{2}}=1+\tan (n s)
$$

Proof. If we take the derivative of equation (3.27) and from the equation (2.7) we get

$$
\left(T_{B}\right)_{\zeta_{2}} \cdot \frac{d s^{*}}{d s}=\frac{1}{\sqrt{2}}\left(T_{B}-\tan (n s) T_{B}\right)
$$

if we take the norm in equation (3.28), $\frac{d s^{*}}{d s}=\frac{1-\tan (n s)}{\sqrt{2}}$. We obtain the tangent of $\zeta_{2}(s)$-Smarandahce curve as in

$$
\left(T_{B}\right)_{2}=T_{B}
$$

The derivative of (3.29) is

$$
\left(T_{B}\right)_{\zeta_{2}}^{\prime} \cdot \frac{d s^{*}}{d s}=-B+\tan (n s)\left(B \wedge T_{B}\right)
$$

From eqnarrays (3.27) and (3.29) we have

$$
\left(B \wedge T_{B}\right)_{\zeta_{2}}=\frac{1}{\sqrt{2}}\left(-B+\left(B \wedge T_{B}\right)\right)
$$

So the geodesic curvature from the equation (2.7) is

$$
K_{g}^{\zeta_{2}}=1+\tan (n s)
$$


Definition 3.21. Let $\zeta=\zeta(s)$ be a curve and $\left\{B, T_{B}, B \wedge T_{B}\right\}$ be Sabban frame of this curve. Then $T_{B}\left(B \wedge T_{B}\right)$-Smarandache curve $\left(\zeta_{3}(s)\right.$-Smarandache curve) is given by

$$
\zeta_{3}(s)=\frac{1}{\sqrt{2}}\left(T_{B}+\left(B \wedge T_{B}\right)\right)
$$

According to equation (2.8) we can parameterize the $\zeta_{3}(s)$-Smarandache curve as in the following form

$$
\zeta_{3}(s)=\frac{1}{\sqrt{2}}\left(\cos (s) \sin (n s)-n \sin (s) \cos (n s)+\frac{n}{m} \sin (s), \sin (s) \sin (n s)+n \cos (s) \cos (n s)-\frac{n}{m} \cos (s), \frac{n}{m} \cos (n s)+n\right) .
$$

The shape of this curve is given in Figure (3.11)
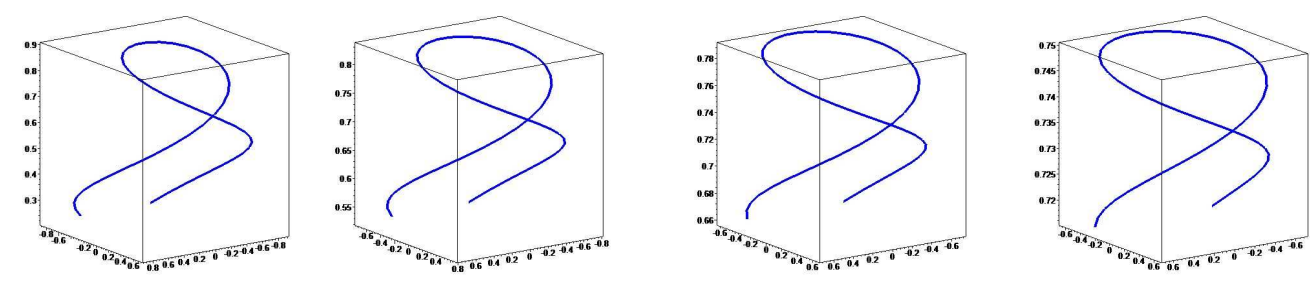

Figure 3.11: $T_{B}\left(B \wedge T_{B}\right)$-Smarandache Curve , $m=\frac{1}{3}, \frac{1}{5}, \frac{1}{8}, \frac{1}{16}$ and $s=[-5,5]$

Theorem 3.22. The geodesic curvature $K_{g}^{\zeta_{3}}$ according to $\zeta_{3}(s)$-Smarandache curve is

$$
K_{g}^{\zeta_{3}}=\frac{1}{\left(1+2(\tan (n s))^{2}\right)^{\frac{5}{2}}}\left(2 \tan (n s) \chi_{22}-\chi_{23}+\chi_{24}\right),
$$

where the coefficients $\chi_{22}, \chi_{23}, \chi_{24}$ are

$$
\begin{aligned}
& \chi_{22}=2 \tan (n s) \tan (n s)^{\prime}+\tan (n s)+2 \tan ^{3}(n s), \\
& \chi_{23}=-1-\tan (n s)^{\prime}-3 \tan ^{2}(n s)-2 \tan ^{4}(n s), \\
& \chi_{24}=-\tan ^{2}(n s)+2 \tan (n s)^{\prime}-2 \tan ^{4}(n s) .
\end{aligned}
$$

Proof. If we take the derivative of equation (3.30) and from the equation (2.7) we get

$$
\left(T_{B}\right)_{\zeta_{3}} \cdot \frac{d s^{*}}{d s}=\frac{1}{\sqrt{2}}\left(-B-\tan (n s) T_{B}+\tan (n s)\left(B \wedge T_{B}\right)\right),
$$

if we take the norm of eqnarray (3.31) we have

$$
\frac{d s^{*}}{d s}=\frac{1}{\sqrt{2}} \sqrt{1+2 \tan ^{2}(n s)}
$$

We obtain the tangent of $\zeta_{3}(s)$-Smarandahce curve as in

$$
\left(T_{B}\right)_{\zeta_{3}}=\frac{1}{\sqrt{1+2 \tan ^{2}(n s)}}\left(-B-\tan (n s) T_{N}+\tan (n s)\left(B \wedge T_{B}\right)\right)
$$

The derivative of (3.32) is

$$
\left(T_{B}\right)_{\zeta_{3}}^{\prime} \cdot \frac{d s^{*}}{d s}=\frac{\sqrt{2}}{\left(1+2 \tan ^{2}(n s)\right)^{2}}\left(\chi_{22} B+\chi_{23} T_{B}+\chi_{24}\left(B \wedge T_{B}\right)\right)
$$

From equations (3.30) and (3.32) we have

$$
\left(B \wedge T_{B}\right)_{\zeta_{3}}=\frac{1}{\sqrt{2+4 \tan ^{2}(n s)}}\left(2 \tan (n s) B-T_{B}+\left(B \wedge T_{B}\right)\right)
$$

So the geodesic curvature from the equation (2.7) is

$$
K_{g}^{\zeta_{3}}=\frac{1}{\left(1+2(\tan (n s))^{2}\right)^{\frac{5}{2}}}\left(2 \tan (n s) \chi_{22}-\chi_{23}+\chi_{24}\right) .
$$


Definition 3.23. Let $\zeta=\zeta(s)$ be a curve and $\left\{B, T_{B}, B \wedge T_{B}\right\}$ be Sabban frame of this curve. Then $B T_{B}\left(B \wedge T_{B}\right)$-Smarandache curve $\left(\zeta_{4}(s)\right.$-Smarandache curve) is given by

$$
\zeta_{4}(s)=\frac{1}{\sqrt{3}}\left(B+T_{B}+\left(B \wedge T_{B}\right)\right)
$$

According to equation (2.8) we can parameterize the $\zeta_{4}(s)$-Smarandache curve as in the following form

$$
\begin{aligned}
\zeta_{4}(s)= & \frac{1}{\sqrt{3}}\left(-\cos (s)(\cos (n s)-\sin (n s))-n \sin (s)(\cos (n s)+\sin (n s))+\frac{n}{m} \sin (s),-\sin (s)(\cos (n s)-\sin (n s))+n \cos (s)(\cos (n s)\right. \\
& \left.+\sin (n s))-\frac{n}{m} \cos (s), \frac{n}{m}(\cos (n s)+\sin (n s))+n\right) .
\end{aligned}
$$

The shape of this curve is given in Figure (3.12)
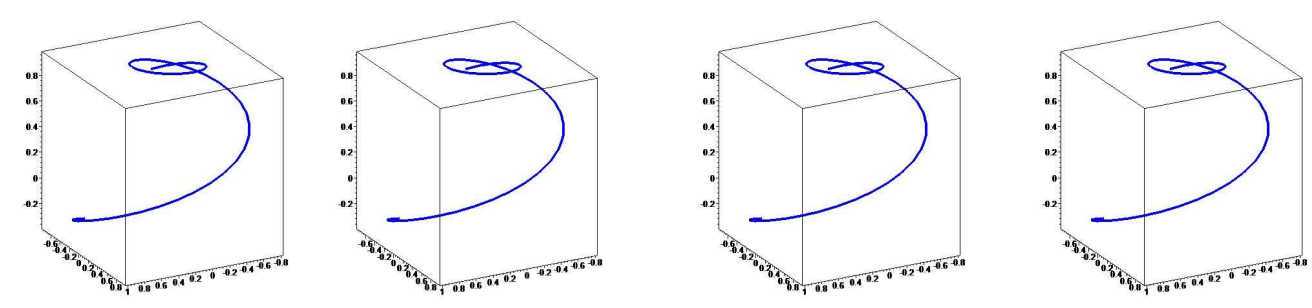

Figure 3.12: $B T_{B}\left(B \wedge T_{B}\right)$-Smarandache Curve, $m=\frac{1}{3}, \frac{1}{5}, \frac{1}{8}, \frac{1}{16}$ and $s=[-5,5]$

Theorem 3.24. The geodesic curvature $K_{g}^{\zeta_{4}}$ according to $\zeta_{4}(s)$-Smarandache curve is

$$
K_{g}^{\zeta_{4}}=\frac{\left(\chi_{25}(2 \tan (n s)-1)+\chi_{26}(-1-\tan (n s))+\chi_{27}(2-\tan (n s))\right)}{\left(4 \sqrt{2}\left(1-\tan (n s)+\tan ^{2}(n s)\right)^{2}\right)^{\frac{5}{2}}},
$$

where the coefficients $\chi_{25}, \chi_{26}, \chi_{27}$ are

$$
\begin{aligned}
\chi_{25}= & -\tan (n s)^{\prime}+2 \tan (n s) \tan (n s)^{\prime}-2+4 \tan (n s)-4 \tan ^{2}(n s) \\
& +2 \tan ^{3}(n s), \\
\chi_{26}= & -\tan (n s)^{\prime}-\tan (n s) \tan (n s)^{\prime}-2-4 \tan ^{2}(n s)+2 \tan (n s) \\
& +2 \tan ^{3}(n s)-2 \tan ^{4}(n s), \\
\chi_{27}= & \tan (n s) \tan (n s)^{\prime}+2 \tan (n s)-4 \tan ^{2}(n s)+2 \tan (n s)^{\prime}+4 \tan ^{3}(n s) \\
& -2 \tan ^{4}(n s) .
\end{aligned}
$$

Proof. If we take the derivative of equation (3.33) and from the equation (2.7) we have

$$
\left(T_{B}\right)_{\zeta_{4}} \cdot \frac{d s^{*}}{d s}=\frac{1}{\sqrt{3}}\left(-B+(1-\tan (n s)) T_{B}+\tan (n s)\left(B \wedge T_{B}\right)\right)
$$

if we take the norm of equation (3.34)

$$
\frac{d s^{*}}{d s}=\frac{1}{\sqrt{3}} \sqrt{2\left(1-\tan (n s)+\tan ^{2}(n s)\right)}
$$

We obtain the tangent of $\zeta_{4}(s)$-Smarandahce curve as in

$$
\left(T_{B}\right)_{\zeta_{4}}=\frac{1}{\sqrt{2\left(1-\tan (n s)+\tan ^{2}(n s)\right)}}\left(-B+(1-\tan (n s)) T_{B}+\tan (n s)\left(B \wedge T_{B}\right)\right) .
$$

The derivative of (3.35) is

$$
\left(T_{B}\right)_{\zeta_{3}}^{\prime} \cdot \frac{d s^{*}}{d s}=\frac{\sqrt{3}}{4\left(1-\tan (n s)+\tan ^{2}(n s)\right)^{2}}\left(\chi_{25} B+\chi_{26} T_{B}+\chi_{27}\left(B \wedge T_{B}\right)\right) .
$$

From equations (3.33) and (3.35) we have

$$
\left(B \wedge T_{B}\right)_{\zeta_{4}}=\frac{\left((-1+2 \tan (n s)) B+(-1-\tan (n s)) T_{B}+(2-\tan (n s))\left(B \wedge T_{B}\right)\right)}{\sqrt{6\left(1-\tan (n s)+\tan ^{2}(n s)\right)}} .
$$

So the geodesic curvature from the equation (2.7) is

$$
K_{g}^{\zeta_{4}}=\frac{\left(\chi_{25}(2 \tan (n s)-1)+\chi_{26}(-1-\tan (n s))+\chi_{27}(2-\tan (n s))\right)}{\left(4 \sqrt{2}\left(1-\tan (n s)+\tan (n s)^{2}\right)^{2}\right)^{\frac{5}{2}}} .
$$




\section{Acknowledgement}

This research is supported by Ordu University Scientific Research Projects Coordination Unit (BAP). Project Number: B-1829

\section{References}

[1] E. Salkowski, Zur transformation von raumkurven, Math. Ann., 4(66) (1909), 517-557.

[2] J. Monterde, Salkowski curves revisited: A family of curves with constant curvature and non-constant torsion, Comput. Aided Geom. Design, 26 (2009), $271-278$.

[3] A. T. Ali, Spacelike Salkowski and anti-Salkowski curves with a spacelike principal normal in Minkowski 3-space, Int. J. Open Problems Compt. Math., 2(3) (2009), 451-460.

[4] A. T. Ali, Timelike Salkowski curves in Minkowski E ${ }_{1}^{3}$, JARDCS, 2(1) (2010), 17-26.

[5] S. Gür, S. Şenyurt, Frenet vectors and geodesic curvatures of spheric indicators of Salkowski curve in E3, Hadronic J., 33(5) (2010), 485.

[6] S. Şenyurt, B. Öztürk, Smarandache curves of Salkowski curve according to Frenet frame, Turk. J. Math. Comput. Sci., 10(2018), $190-201$.

[7] S. Şenyurt, B. Öztürk, Smarandache curves of anti-Salkowski curve according to Frenet frame, Proceedings of the International Conference on Mathematical Studies and Applications (ICMSA 2018), (2018), 132-143.

[8] M. Turgut, S. Yilmaz, Smarandache curves in Minkowski spacetime, Int. J. Math. Comb., 3 (2008), 51-55.

[9] A. T. Ali, Special Smarandache curves in the Euclidean space, Int. J. Math. Comb., 2 (2010), 30-36.

[10] S. Şenyurt, A. Çalışkan, $N^{*} C^{*}$-Smarandache curves of Mannheim curve couple according to Frenet frame, Int. J. Math. Comb., 1 (2015), 1-15.

[11] S. Şenyurt, B. Öztürk, anti-Salkowski eğrisine ait Frenet vektörlerinden elde edilen Smarandache eğrileri, Karadeniz 1. Uluslararası Multidisipliner Çalışmalar Kongresi, Giresun, (2019), 463-471.

[12] J. Koenderink, Solid Shape, MIT Press, Cambridge, MA, 1990.

[13] K. Taşköprü, M. Tosun, Smarandache curves according to Sabban frame on S2, Bol. Soc. Paran. Mat., 32 (2014), $51-59$.

[14] M. P. Do Carmo, Differential Geometry of Curves and Surfaces: Revised and Updated Second Edition, Courier Dover Publications, 2016. 